\title{
Magnetic Field Effects in the Pseudogap Phase: A Competing Energy Gap Scenario for Precursor Superconductivity
}

\author{
Ying-Jer Kao ${ }^{1}$, Andrew P. Iyengar ${ }^{1}$, Qijin Chen ${ }^{2}$ and K. Levin ${ }^{1}$ \\ ${ }^{1}$ James Franck Institute and Department of Physics, University of Chicago, Chicago, Illinois 60637 \\ ${ }^{2}$ National High Magnetic Field Laboratory, Tallahassee, Florida 32310
}

(October 28, 2018)

\begin{abstract}
We study the sensitivity of $T_{c}$ and the pseudogap onset temperature , $T^{*}$, to low fields, $H$, using a BCS-based approach extended to arbitrary coupling. We find that $T^{*}$ and $T_{c}$, which are of the same superconducting origin, have very different $H$ dependences. This is due to the pseudogap, $\Delta_{p g}$, which is present at the latter, but not former temperature. Our results for the coherence length $\xi$ fit well with existing experiments. We predict that very near the insulator $\xi$ will rapidly increase.
\end{abstract}

PACS numbers: 74.25.Ha, 74.60.Ec, 74.25.-q

cond-mat/0103614

One of the central questions in understanding the underdoped cuprates is the extent to which the superconducting phase is described by BCS theory. Recent experiments 13 indicate that the pseudogap persists below $T_{c}$ in the underlying normal density of states. Thus, the fermionic excitation gap $\Delta$ is to be distinguished from the order parameter $\Delta_{s c}$. These two energy gaps mirror a distinction between the two temperatures $T^{*}$ (the pseudogap onset), and $T_{c}$ (the superconducting transition), which behave differently as a function of hole concentration $x$ as well as of magnetic field, $H 46$. Indeed, $T^{*}$ and $T_{c}$ are, respectively, weakly and strongly dependent on $H$ in the well-established pseudogap regime. Moreover, the distinction between these temperature and energy scales has been frequently cited 2 , 1 as evidence that they have different physical origins.

In this paper we provide a counter argument to this widely stated inference by demonstrating that these crucial magnetic field effects in the pseudogap phase, are entirely compatible with superconductivity as origin for both $T^{*}$ and $T_{c}$. Our approach is based on an extended version of BCS theory, in which the attractive coupling $g$ is contemplated to be strong enough so that pairs begin to form at a higher femperature $T^{*}$ than the $T_{c}$ at which they Bose condenset 8 . We have shown 911 that as a necessary fpnsequence $\Delta \neq \Delta_{s c}$. Moreover, our work has emphasized 12.13 that a (pseudo)gap in the fermionic spectrum at $T_{c}$ is deleterious for superconductivity. Thus, as observed experimentally, as a function of decreasing $x, T_{c}$ decreases as the pseudogap or $T^{*}$ grows.

A calculation of the field dependence of $T_{c}$ (i.e., $H_{c 2}$ ) is an important problem in its own right. (i) This is the only way to provide a precise interpretation of the "coherence length" $\xi$, which we demonstrate here is very different from that of BCS theory 14 . (ii) An analysis of $H_{c 2}$ is tantamount to arriving at a reformulation of the microscopically deduced GinzburgLandau (GL) free energy up to quadratic terms, which must necessarily incorporate the presence of a non-zero pseudo-

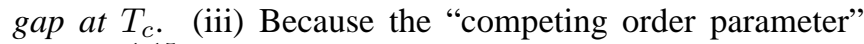
scenarid 1,15 also addresses the observation that $\Delta \neq \Delta_{s c}$, as well as the competing $x$ dependences of $T_{c}$ and $T^{*}$, magnetic field effects may provide a unique testing ground for distinguishing between these two scenarios.
Indeed there is a rather close similarity in the structure of our zero-field theory to the phenomenology deduced from thermodynamical data by Loram et at 6 . Our mean field calculations $9-11$ show that the gap equation for $T \leq T_{c}$ reduces to the usual BCS form, but with a new quasi-particle dispersion

$$
E_{\mathbf{k}}=\sqrt{\epsilon_{\mathbf{k}}^{2}+\left(\Delta_{s c}^{2}+\Delta_{p g}^{2}\right) \varphi_{\mathbf{k}}^{2}}=\sqrt{\epsilon_{\mathbf{k}}^{2}+\Delta^{2} \varphi_{\mathbf{k}}^{2}} .
$$

Here $\varphi_{\mathbf{k}}$ is associated with the pairing symmetry. It follows from this that the larger is $\Delta_{p g}\left(T_{c}\right)$, the lower the transition temperature $T_{c}$. In contrast to the work in Refs. 16 and 15 , here $\Delta_{p g}(T)$, is determined self-consistently and derives from the presence of a strong pairing attraction. Moreover, this pseudogap, $\Delta_{p g}$, persists below $T_{c}$.

The conclusions of this paper are relatively straightforward and we begin with a simple intuitive argument to address $H_{c 2}$. Consider the Ginzburg-Landau free energy functional near $T_{c}$ to quadratic order in $\Delta_{s c}$, in a finite field

$$
F \sim\left(\tau_{0}(T)+\eta^{2}\left(\frac{\nabla}{i}-\frac{2 e \mathbf{A}}{c}\right)^{2}\right)\left|\Delta_{s c}\right|^{2} .
$$

Here $\tau_{0}$ describes how the system approaches the critical point with varying temperature, and $\eta^{2}$ is the stiffness against spatial variations of the order parameter. The mean-field behavior of $\tau_{0}$ near $T_{c}$ yields $\tau_{0}(T)=\bar{\tau}_{0}\left(1-T / T_{c}\right)$. It follows that

$$
-\left.\frac{1}{T_{c}} \frac{d T_{c}}{d H}\right|_{H=0}=\frac{2 \pi}{\Phi_{0}} \xi^{2}=\frac{2 \pi}{\Phi_{0}} \frac{\eta^{2}}{\bar{\tau}_{0}} .
$$

where $\xi$ is the zero temperature coherence length. A rough extrapolation yields $H_{c 2}(0) \approx \Phi_{o} /\left(2 \pi \xi^{2}\right)$. In the small $g$ (i.e., BCS) case $\bar{\tau}_{0}=N(0)$, the density of states per spin at the Fermi surface, and $\eta^{2}=N(0) 7 \zeta(3) / 48 \pi^{2}\left(v_{F} / T_{c}\right)^{2}$. The squared coherence length $\xi_{B C S}^{2}=7 \zeta(3) / 48 \pi^{2}\left(v_{F} / T_{c}\right)^{2}$ is determined by the stiffness $\eta^{2}$ with $\bar{\tau}_{0}$ cancelling the density of states.

In contrast, in the strong coupling case the pseudogap modifies the fermionic quasiparticle dispersion through a replacement of $\epsilon_{\mathbf{k}}$ by $E_{\mathbf{k}}$ and thereby suppresses $\bar{\tau}_{0}$. Moreover, the stiffness $\eta^{2}$ (which is relatively insensitive to the energy scale 
of the pseudogap), decreases due to the diminishing pair size. There is, thus, a competition between the numerator and denominator in Eq. (3). The decrease in $\bar{\tau}_{0}$ dominates at sufficiently large coupling $g$, resulting in an extended flat region followed by an eventual growth of $\xi^{2}$ with increasing $g$. The latter reflects the approach to the ideal "boson" limit, where $T_{c}$ is suppressed 17 to zero at any $H \neq 0$.

Next, we provide a microscopic derivation of the parameters in Eq. (3) (and a related counterpart for $T^{*}$ ). A central theme in our paper is that both the field sensitivity of $T_{c}$ and of $T^{*}$ in small $H$ can be studied through the zerofield normal state pair propagator, or the inverse $t$-matrix $t^{-1}(Q)=1 / g+\chi(Q)$, where $Q=(\mathbf{q}, \Omega)$ is a four-vector. The coefficients in Eq. (2) will be shown to arise from an expansion of $t^{-1}$ in the momentum components perpendicular to the field (indexed by $i, j=x, y$ ) as

$$
\tau_{0}=\frac{1}{g}+\chi(\mathbf{0}, 0), \eta^{2}=\left.\frac{1}{2} \sqrt{\operatorname{det}\left[\partial_{q_{i}} \partial_{q_{j}} \chi(Q)\right]}\right|_{Q=0}
$$

where we have generalized from Eq. (2) to include possible anisotropy.

A proper metivation for our choice of $\chi(Q)$ is essential. The formalism 1011 in this paper combines a Green's function decoupling scheme 18 with a generalization of the BCS ground state wavefunction 19 . This formalism allows for Fermi- and non-Fermi-liquid (i.e., $\Delta_{p g}\left(T_{c}\right) \neq 0$ ) based superconductivity, according to the size of $g$, with self consistently determined chemical potential $\mu$. In the present paper all technical issues of this decoupling scheme can be simply by-passed, and the results obtained are not only intuitive, but rather general. All that is needed here is the observation that the pair susceptibility $\chi(\mathbf{q}, \Omega)=\chi(Q)=\sum_{K} G(K) G_{0}(Q-K) \varphi_{\mathbf{k}-\mathbf{q} / 2}^{2}$. Thus

$$
\begin{aligned}
& \chi(\mathbf{q}, 0)=\sum_{\mathbf{k}} \varphi_{\mathbf{k}-\mathbf{q} / 2}^{2} \times \\
& {\left[\frac{1-f\left(E_{\mathbf{k}}\right)-f\left(\epsilon_{\mathbf{k}-\mathbf{q}}\right)}{E_{\mathbf{k}}+\epsilon_{\mathbf{k}-\mathbf{q}}} u_{\mathbf{k}}^{2}-\frac{f\left(E_{\mathbf{k}}\right)-f\left(\epsilon_{\mathbf{k}-\mathbf{q}}\right)}{E_{\mathbf{k}}-\epsilon_{\mathbf{k}-\mathbf{q}}} v_{\mathbf{k}}^{2}\right] .}
\end{aligned}
$$

Here $u_{\mathbf{k}}^{2}$ and $v_{\mathbf{k}}^{2}$ are the usual BCS coherence factors, and $\varphi_{\mathbf{k}}^{2}=\left(1+\left(k / k_{0}\right)^{2}\right)^{-1}$, or $\left(\cos \left(k_{x} a\right)-\cos \left(k_{y} a\right)\right)^{2}$, for $s-$ wave pairing in 3D jellium or $d$-wave pairing on a quasi- $2 \mathrm{D}$ lattice, respectively. That there is one full Green's function $(G)$ along with one bare Green's function $\left(G_{0}\right)$, reflects the structure of the BCS gap equation, which introduces 18 this $\chi(Q)$ form, (with integrand proportional to the usual Gor'kov $F$ function). All numerical calculations in this paper are based on Eqs. (1), (4), and (5), given $\Delta_{p g} \equiv \Delta_{p g}\left(T_{c}\right)$. Although here we proceed more self consistently, ow analytical scheme for computing the various energy scales 13 can be by-passed, if $\Delta_{p g}$ and $T_{c}$ are pre-determined, e.g., fitted to cuprate experiments.

We next turn to $T^{*}$, where the Fermi liquid begins to-preak down; this is associated with the onset of a resonance 20 21 in $t(Q)$, as $g$ becomes sufficiently large. Detailed numerics 21,12 based on the coupled Green's function equations show that to a good first order approximation this resonance temperature can be deduced from the condition $1 / g+\chi_{0}(\mathbf{0}, 0)=0$, where $\chi_{0}$ is given by Eq. (5) with $\epsilon_{k}$ substituted for $E_{\mathbf{k}}$. Indeed, quite generally, at $T \geq T^{*}$, the $t$-matrix can be well approximated by using $\chi_{0}(Q)$ in place of $\chi(Q)$.

Magnetic field effects can be readily included into our formalism. We begin with a derivation of $T^{*}(H)$ to linear order in $H$. Our Hamiltonian consists of the field dependent kinetic energy term along with the usual two-body pairing interaction of strength $V_{\mathbf{k}, \mathbf{k}^{\prime}}=g \varphi_{\mathbf{k}} \varphi_{\mathbf{k}^{\prime}}^{*}$ where we now include interactions between pairs of non-zero net momentum. Fluctuations of pairs with finite momentum $(\mathbf{q}, \mathbf{k})$ are characterized by the correlation function

$$
D\left(q, k ; q^{\prime}, k^{\prime}\right)=\left\langle\mathcal{T} b(q, k, \tau) b^{\dagger}\left(q^{\prime}, k^{\prime}, \tau^{\prime}\right)\right\rangle,
$$

where $b(q, k, \tau)=e^{(\mathcal{H}-\mu N) \tau} c_{q / 2-k} c_{q / 2+k} e^{-(\mathcal{H}-\mu N) \tau}$.

Summing ladder diagrams leads to a Dyson equation $D=$ $G_{0} G_{0}-G_{0} G_{0} V D$ with solution

$$
\sum_{k, k^{\prime}} D \varphi_{k}^{*} \varphi_{k^{\prime}}=\frac{\widehat{\chi_{0}}}{1+g \widehat{\chi_{0}}}
$$

where $\widehat{\chi_{0}}=G_{0} G_{0}$ is the counterpart pair susceptibility for $H \neq 0$, and the field-dependence of the bare electron propagator $G_{0}$ is implemented using the semiclassical phase approximation 22 , elevating both $\widehat{\chi_{0}}$ and $\sum_{k, k^{\prime}} D \varphi_{k}^{*} \varphi_{k^{\prime}}$ to integral operator 23 whose eigenvalues satisfy Eq. (6). This approximation allows the calculation of the eigenvalues of $\widehat{\chi_{0}}$ from the zero-field pair susceptibility $\chi_{0}$ in the regime $T \gg e H / m c$. The field-induced relative phase shift between electrons in a pair renormalizes the interaction $V_{\mathbf{k}, \mathbf{k}^{\prime}}$, but the effect is quadratic in $H$ and is therefore ignored here. The pairing resonance temperature $T^{*}$ is defined by the appearance of an eigenvalue $\Pi_{0}=-g^{-1}$ of $\widehat{\chi_{0}}$, which causes Eq. (6) to diverge. We define parameters $\eta^{* 2}$ and $\tau_{0}^{*}=\bar{\tau}_{0}^{*}\left(1-T / T^{*}\right)$ analogous to those which appear in Eq. (4) and obtain

$$
g^{-1}+\Pi_{0}=\tau_{0}^{*}+\eta^{* 2} \cdot \frac{2 e}{c} H=0
$$

which defines $T^{*}(H)$ to linear order in $H$. The slope of $T^{*}(H)$ at $H=0$ is

$$
-\left.\frac{1}{T^{*}} \frac{d T^{*}}{d H}\right|_{H=0}=\frac{\eta^{* 2}}{\bar{\tau}_{0}^{*}} \frac{2 \pi}{\Phi_{0}}
$$

which leads to the associated "coherence length" $\xi^{* 2}=$ $\eta^{* 2} / \bar{\tau}_{0}^{*}$. The stiffness $\eta^{* 2}$ can be explicitly evaluated using the zero-field pair susceptibility, and

$$
\begin{aligned}
\bar{\tau}_{0}^{*}=\sum_{\mathbf{k}} \varphi_{\mathbf{k}}^{2} & {\left[-f^{\prime}\left(\epsilon_{\mathbf{k}}\right)+\frac{d \mu}{d T} \frac{T}{\epsilon_{\mathbf{k}}} .\right.} \\
& \left.\left(\frac{1-2 f\left(\epsilon_{\mathbf{k}}\right)}{2 \epsilon_{\mathbf{k}}}+f^{\prime}\left(\epsilon_{\mathbf{k}}\right)\right)\right]_{T=T^{*}}
\end{aligned}
$$

Here we have included a contribution from the temperature dependence of $\mu$. In the weak coupling case, the chemical 


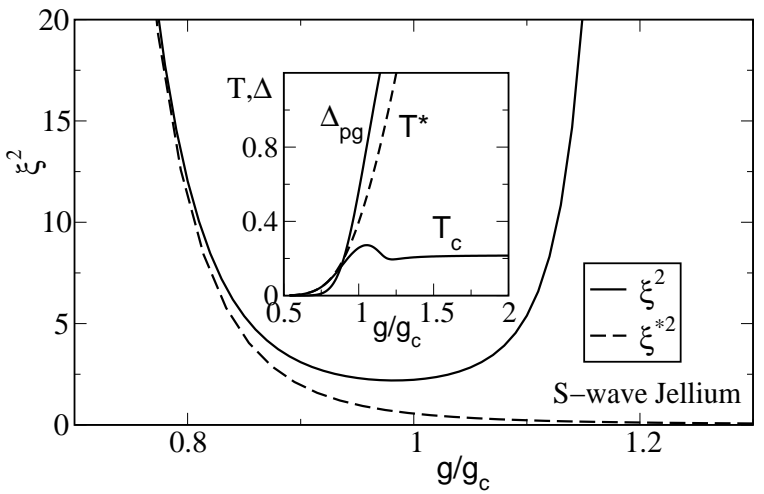

FIG. 1. Coherence lengths $\xi$ (for $T_{c}$, solid) and $\xi^{*}$ (for $T^{*}$, dashed) with variable coupling $g$ for $s$-wave jellium; here $g_{c} \equiv-4 \pi / m k_{0}$ and units are $k_{F}^{-1}$. See Eqs. (3) and (8. Inset shows the zero field behavior of $T^{*}, T_{c}$, and $\Delta_{p g}$ from Ref $\mathbb{E}$, where $T$ and $\Delta$ are in units of $E_{F}$.

potential is pinned at $E_{F}$, and we recover the (s-wave) BCS limit $\bar{\tau}_{0}^{*}=-\sum_{\mathbf{k}} f^{\prime}\left(\epsilon_{\mathbf{k}}\right) \varphi_{\mathbf{k}}^{2} \approx N(0) \varphi_{k_{F}}^{2} \approx N(0)$.

The dashed line in Fig. 1 is a plot of the slope of $T^{*}$ with $H$, as a function of the coupling $g$, for the case of $s$-wave jellium. This should be compared with the inset, from Ref. 13 , which illustrates the suppression of $T_{c}$ by $\Delta_{p g}$ for all $g$ in the fermionic regime; (ultimately, when $\mu$ becomes negative, $T_{c}$ starts to increase again). When pseudogap effects are weak, $T^{*}$ is essentially the same as $T_{c}$ and depends strongly on $H$. A stronger pairing interaction $g$ causes $\Delta_{p g}$ to increase and the pair size to decrease; the latter effect diminishes the stiffness and causes $T^{*}$ to be weakly field dependent24.

We turn next to $T_{c}(H)$ and note that a solution of the coupled equations of motion 18 (as was done in the zero field case) appears prohibitively difficult. Nevertheless, based on the above observations that (i) in zero field $T^{*}$ scales father well with $\Delta_{p g}$ (both theoretically 13 and experimentallyt,) and (ii) that $T^{*}$ is very weakly field dependent in the well-established pseudogap regime, we infer that $\Delta_{p g} \equiv \Delta_{p g}\left(T_{c}\right)$ is weakly $H$ dependent. This assumption, along with the semi-classical phase approximation for the full Green's function, are the only essential assumptions made here. The weak $H$ dependence in $\Delta_{p g}$ appears compatible with experiment 6.3 and underlies a GL formulation (Eq. (2)) in which only the superconducting order parameter is coupled to the magnetic field. In this way, for the purposes of computing $H_{c 2}$, the pseudogap enters as a relatively rigid band structure effect, which is accounted for by introducing the full pair susceptibility into the standard $H_{c 2}$ formalism 23. This approach necessarily yields the correct $H=0$ result for $T_{c}$.

It also leads naturally to Eq. (4), from which one deduces a rather complicated expression (not shown) for $\eta^{2}$ along with

$$
\bar{\tau}_{0}=-\sum_{\mathbf{k}} \varphi_{\mathbf{k}}^{2} f^{\prime}\left(E_{\mathbf{k}}\right)
$$

where we have omitted contributions from the temperature dependence of $\mu$ and $\Delta_{p g}$ near $T_{c}$. Eq. 10) contains the essential physics introduced by the pseudogap. The summation essentially measures the $E=0$ density of states which

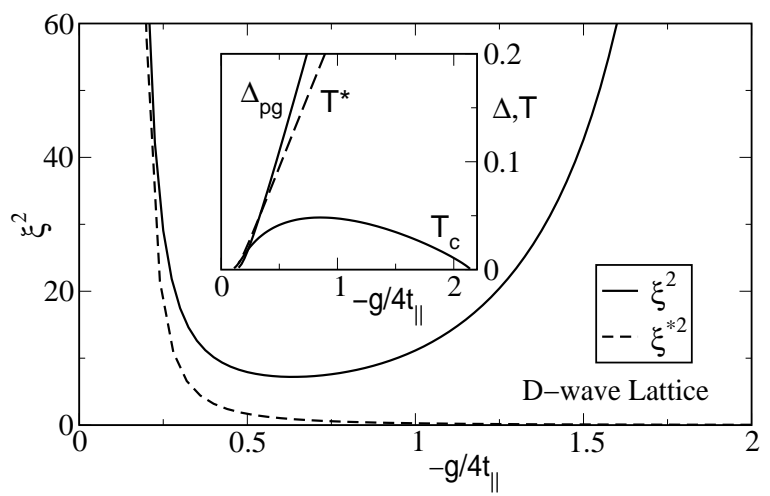

FIG. 2. Coherence lengths at $T_{c}$ (solid) and $T^{*}$ (dashed) versus variable coupling $g$ for a $d$-wave lattice at density $n=0.85$. $\xi$ is units of $a$. The inset plots the zero field energy scales from Ref. 13 , and $T, \Delta$ are in units of $4 t_{\|}$.

is depleted by the pseudogap at strong coupling, leading to $\bar{\tau}_{0} \sim e^{-\Delta_{p g} / T_{c}}$. Moreover, we have shown analytically that the neglected terms further suppress $\bar{\tau}_{0}$, and, therefore, do not qualitatively change our results.

Figures 1 and 2 indicate how the characteristic low-field slopes behave as a function of coupling $g$, for the $s$-wave (jellium) and $d$-wave (lattice) cases, respectively. For the latter, the bare band dispersion was presumed to be $\epsilon_{\mathbf{k}}=2 t_{\|}(2-$ $\left.\cos \left(k_{x} a\right)-\cos \left(k_{y} a\right)\right)-2 t_{\perp}\left(1-\cos \left(k_{\perp} d\right)\right)-\mu$, where $a$ is the lattice constant in the plane and $d$ is the distance between layers. The insets in each figure summarize the zero field results for $T_{c}, T^{*}$, and $\Delta_{p g}$, calculated elsewhere 13 . It should be noted from the insets that there is an extended regime of coupling constants over which BCS behavior $\left(\Delta_{p g}=0\right.$, and $\left.T_{c}=T^{*}\right)$ is obtained. However, beyond a "critical" coupling (corresponding roughly to where bound states of the isolated pair occur,) the pseudogap becomes non-zero, and $T_{c}$ is differentiated from $T^{*}$.

It is clear from the figures that the slopes of $T^{*}$ and $T_{c}$ are identical at weak coupling and become progressively more distinct as the coupling is increased. The two associated stiffness parameters decrease with coupling in a similar way, but the field dependence of $T_{c}$ is enhanced by the strong suppression of $\bar{\tau}_{0}$ relative to $\bar{\tau}_{0}^{*}$. The competition between the numerator and denominator in Eq. (3) leads to a length scale $\xi^{2}$ which is relatively constant over a rather wide range of moderate $g$; then at sufficiently strong coupling $\xi^{2}$ begins to increase due to the reduction in the density of states $\left(\bar{\tau}_{0}\right)$ associated with the growth of the pseudogap.

The key role of $\bar{\tau}_{0}$ in determining the squared-coherence length $\xi^{2}$ highlights the fact that at strong coupling, the various length scales of the system must be carefully distinguished even though they are identical in BCS theory. This is illustrated in Figs. 3 (a) and (b) for the $s$ - and $d$-wave cases, respectively, in which we compare normalized stiffness, $\bar{\eta}=$ $\eta / \sqrt{N(0)}$, with the BCS coherence length at $T_{c}$, and $\xi$, the calculated coherence length at $T_{c}$. The density of states effect is evident in both the $s$ - and $d$-wave cases, as the upturn of $\xi$ at strong coupling contrasts sharply with the tapering of the stiffness $\bar{\eta}$. Note that $\xi_{B C S}$ exhibits unusual structure in 

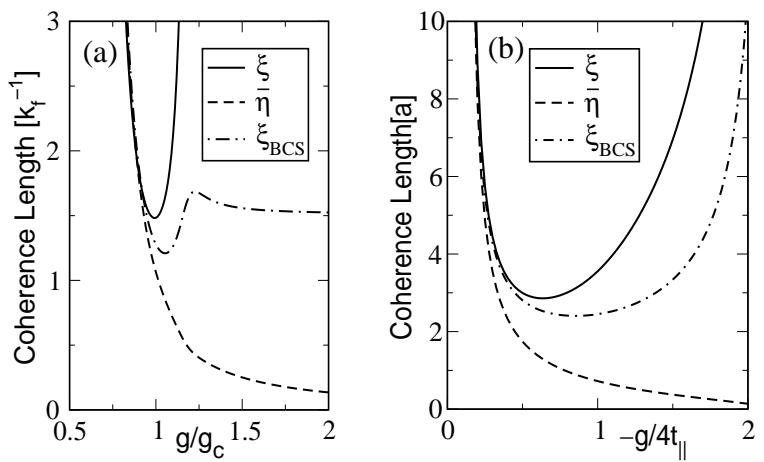

FIG. 3. Coupling constant dependence of the key length scales: the calculated coherence length $\xi$ obtained from $H_{c 2}$ (solid), $\bar{\eta}$ (the normalized stiffness; dashed), and the BCS coherence length ( $\propto v_{F} / T_{c}$; dot-dashed), for the $s$-wave jellium case (a) and the $d$-wave lattice case (b).

Fig. B(a) which arises from the non-monotonic behavior of $T_{c}$ evident in the inset of Fig. 1. In Fig. 3(b), the divergence of $\xi_{B C S}$ at large $g$ is "accidental" and derives from the vanishing of $T_{C}$ which arises from pair-localizationt 3 (see Fig. 2, inset). BCS relations, therefore, are very misleading when one tries to infer the behavior of $\xi$ at strong coupling from other length scales in the system.

In order to map the coupling $g$ onto hole concentration $x$, we introduce an $x$-dependent hopping matrix element $t_{\|}(x)=$ $t_{0} x$ associated with the Mott transition 2 . We presume in the absence of more microscopic information that $g$ is $x$ independent, leaving one free parameter in our theory $g / t_{0}$, chosen to optimize a fit to the phase diagram (Fig. A, inset.) One could, alternatively, bypass all assumptions concerning Mott insulator physics, if one instead used experimental data in place of the calculations in the inset. The resulting behavior of $\xi$ and $\xi^{*}$ (Fig. (4) would be essentially the same. Over most of the range of $x, \xi$ is relatively constant, as seems to be observed experimentally, and its magnitude is within a factor of two or three of experimen 25. As in experiment, $T^{*}$ is foupd to be less field sensitive in the underdoped than overdoped regimes. As the insulator is approached, $\xi$ rapidly increases 26 while $\xi^{*}$ continues to decrease. We have thus demonstrated that the different observed field dependences of $T^{*}$ and $T_{c}$ (for both under- and over-doped cuprates) are contained in our theory, in which the pseudogap is associated with precursor superconductivity.

This work was supported by NSF-MRSEC, grant No. DMR-9808595 and by the State of Florida (QC). We acknowledge the hospitality of the ITP, UCSB, where the work was begun, and thank A. Carrington, B. Jankó, V. M. Krasnov, and Z. Tešanović for useful conversations.

Note Added. - After this work was completed we learned of related experimental studies by Shibauchi et al.27 which show strong similarities to our theoretical predictions.

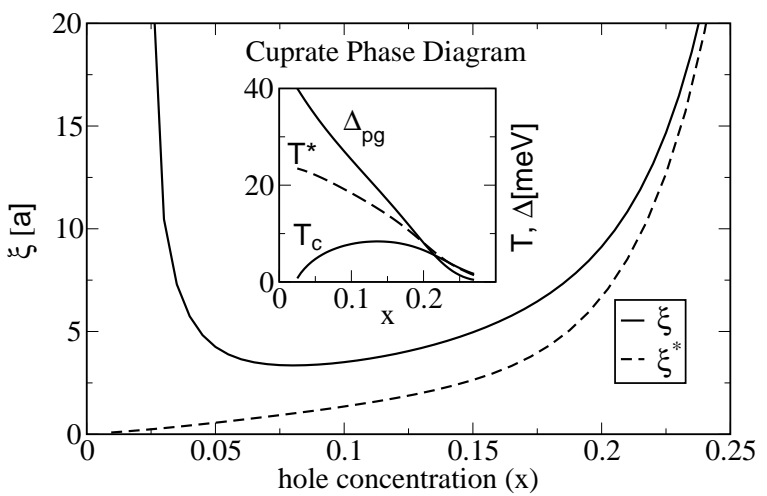

FIG. 4. Magnetic length scales associated with $T_{c}$ and $T^{*}$ as a function of hole concentration in the cuprates. Zero field results from Ref 10 are shown in the inset ( $\Delta_{p g}$ is a factor of 2 smaller than experiment due to our convention for $\varphi_{k}$ ).

${ }^{1}$ J. L. Tallon and J. W. Loram, Physica C 349, 53 (2001).

${ }^{2}$ V. M. Krasnov et al., Phys. Rev. Lett. 84, 5860 (2000).

${ }^{3}$ C. Renner et al., Phys. Rev. Lett. 80, 3606 (1998).

${ }^{4}$ K. Gorny et al., Phys. Rev. Lett. 82, 177 (1999).

${ }^{5}$ G. Q. Zheng et al., Phys. Rev. Lett. 85, 405 (2000).

${ }^{6}$ V. M. Krasnov, A. E. Kovalev, A. Yurgens, and D. Winkler, Phys. Rev. Lett. 86, 2657 (2001).

${ }^{7}$ P. Nozières and S. Schmitt-Rink, J. Low Temp. Phys. 59, 195 (1985).

${ }^{8}$ M. Randeria, Physica B 198, 373 (1994).

${ }^{9}$ I. Kosztin, Q. J. Chen, B. Janko, and K. Levin, Phys. Rev. B 58, R5936 (1998).

${ }^{10}$ Q. J. Chen, I. Kosztin, B. Janko, and K. Levin, Phys. Rev. Lett. 81, 4708 (1998).

${ }^{11}$ Q. J. Chen, I. Kosztin, and K. Levin, Phys. Rev. Lett. 85, 2801 (2000).

12 J. Maly, B. Janko, and K. Levin, Phys. Rev. B 59, 1354 (1999).

${ }^{13}$ Q. J. Chen, I. Kosztin, B. Janko, and K. Levin, Phys. Rev. B 59, 7083 (1999).

${ }^{14} \xi_{B C S}^{2}=7 \zeta(3) / 48 \pi^{2}\left(v_{F} / T_{c}\right)^{2}$ in $3 \mathrm{D}$ and $7 \zeta(3) / 32 \pi^{2}\left(v_{F} / T_{c}\right)^{2}$ in $2 \mathrm{D}$.

${ }^{15}$ S. Chakravarty, R. B. Laughlin, D. K. Morr, and C. Nayak, Phys. Rev. B 63, 094503 (2001).

16 J. W. Loram et al., J. Supercond. 7, 243 (1994).

${ }^{17}$ A. S. Alexandrov, W. H. Beere, V. V. Kabanov, and W. Y. Liang, Phys. Rev. Lett. 79, 1551 (1997);M. R. Schafroth, Phys. Rev. 100, 463 (1955).

${ }^{18}$ L. P. Kadanoff and P. C. Martin, Phys. Rev. 124, 670 (1961).

19 A. J. Leggett, in Modern Trends in the Theory of Condensed Matter (Springer-Verlag, Berlin, 1980), pp. 13-27.

${ }^{20}$ B. Janko, J. Maly, and K. Levin, Phys. Rev. B 56, R11407 (1997).

${ }^{21}$ J. Maly, B. Jankó, and K. Levin, Physica C 321, 113 (1999).

${ }^{22}$ E. Helfand and N. R. Werthamer, Phys. Rev. 147, 288 (1966).

${ }^{23}$ P. A. Lee and M. G. Payne, Phys. Rev. B 5, 923 (1972).

${ }^{24}$ Y. Yanase and K. Yamada, J. Phys. Soc. Jap. 69, 2209 (2000).

${ }^{25}$ H. H. Wen, X. H. Chen, W. L. Yang, and Z. X. Zhao, Phys. Rev. Lett. 85, 2805 (2000).

${ }^{26}$ P. A. Lee and X.-G. Wen, Phys. Rev. Lett. 78, 4111 (1997).

27 T. Shibauchi et al., Phys. Rev. Lett. 86, 5763 (2001). 